\title{
Phase Angle Calculation Dynamics of Type 4 Wind Turbines in RMS Simulations during Severe Voltage Dips.
}

Altin, Müfit; Göksu, Ömer; Sørensen, Poul Ejnar; Morales, Ana; Fortmann, Jens; Buendia, Francisco J.

Published in:

I E T Renewable Power Generation

Link to article, DOI:

10.1049/iet-rpg.2015.0501

Publication date:

2016

Document Version

Peer reviewed version

Link back to DTU Orbit

Citation (APA):

Altin, M., Göksu, Ö., Sørensen, P. E., Morales, A., Fortmann, J., \& Buendia, F. J. (2016). Phase Angle Calculation Dynamics of Type 4 Wind Turbines in RMS Simulations during Severe Voltage Dips. I ET Renewable Power Generation, 10(8), 1069-1186. https://doi.org/10.1049/iet-rpg.2015.0501

\section{General rights}

Copyright and moral rights for the publications made accessible in the public portal are retained by the authors and/or other copyright owners and it is a condition of accessing publications that users recognise and abide by the legal requirements associated with these rights.

- Users may download and print one copy of any publication from the public portal for the purpose of private study or research.

- You may not further distribute the material or use it for any profit-making activity or commercial gain

- You may freely distribute the URL identifying the publication in the public portal 
This article has been accepted for publication in a future issue of this journal, but has not been fully edited. Content may change prior to final publication in an issue of the journal. To cite the paper please use the doi provided on the Digital Library page.

\title{
Phase Angle Calculation Dynamics of Type 4 Wind Turbines in RMS Simulations during Severe Voltage Dips
}

Müfit Altin $^{1 *}$, Ömer Göksu ${ }^{1}$, Poul Sørensen ${ }^{1}$, Ana Morales ${ }^{2}$, Jens Fortmann ${ }^{3}$, Francisco J. Buendia $^{4}$

${ }^{1}$ Wind Energy Dept., Technical University of Denmark (DTU), Roskilde 4000, Denmark

${ }^{2}$ DIgSILENT Ibérica, Madrid 28003, Spain

${ }^{3}$ Hochschule für Technik und Wirtschaft (HTW) Berlin, Germany

${ }^{4}$ Gamesa, Sarriguren, Navarra 31621, Spain

하al@dtu.dk

\begin{abstract}
In order to conduct power system simulations with high shares of wind energy, standard wind turbine models, which are aimed to be generic rms models for a wide range of wind turbine types, have been developed. As a common practice of rms simulations, the power electronic interface of wind turbines is assumed to be ideally synchronized, i.e. grid synchronization (e.g. PLL) is not included in simplified wind turbine models. As will be shown in this paper, this practice causes simulation convergence problems during severe voltage dips and when the loss of synchronism occurs. In order to provide the simulation convergence without adding complexity to the generic models, a first order filtering approach is proposed as a phase angle calculation algorithm in the grid synchronization of the rms type 4 wind turbine models. The proposed approach provides robustness for the simulation of large scale power systems with high shares of wind energy.
\end{abstract}

\section{Introduction}

The share of wind energy in power systems has been increasing considerably for the last decade. Therefore, the need for the power system stability analysis considering wind turbines becomes critical in order to operate the power system within the limits. In the power system analysis, the wind turbines have hitherto been represented by specific models from manufacturers for the Transmission System Operators (TSOs). In order to harmonize the models and increase their availability without dealing confidentiality issues, standards are being developed by the international working groups as IEC61400-27 [1] and WECC [2].

In this paper, the focus is on the full-converter based wind turbine (WT) type, which is covered as a type 4 in the IEC standard [1]. The type 4 model of the IEC standard is a fundamental frequency (rms) positive sequence model specifically developed for the short-term power system stability analysis, such as grid voltage and frequency disturbance events. It includes capabilities such as the grid active power control loop, reactive power and voltage control loops, and the dynamic current injection capability during short-circuit faults. In accordance with the power system stability and the grid code requirements, the analysis of 3-phase (balanced) short-circuit faults has gained great importance. The WTs and wind power plants (WPP) are required to inject primarily reactive current during these faults in order to support the voltage stability [3]. The IEC type $4 \mathrm{WT}$ model, in the reactive power priority mode, has the capability to inject the reactive current proportional to the voltage drop and the active current using the remaining 
This article has been accepted for publication in a future issue of this journal, but has not been fully edited.

Content may change prior to final publication in an issue of the journal. To cite the paper please use the doi provided on the Digital Library page.

capacity, during low voltage faults. This operating mode of primarily reactive and active current injection will be simulated and investigated within this paper for the phase angle calculation during the short-circuit faults.

Fundamental frequency (rms) models are targeted to realize large scale power system analyses as they are simplified in terms of complexity and number of modelling components and hence computationally more efficient than electromagnetic transient (EMT) models, while capturing the sufficient representation of the related dynamics. As a common practice in the rms models, the dynamics of grid synchronization is omitted, such that the converter is modelled as an ideal current source with only a first order transfer function as the representation of its response time [1], [2]. The developed rms WT models have been simulated and validated against field measurements by the WT manufacturers during grid disturbance tests, such as grid faults where the grid voltage drops down to 0.2pu [2]. However, as reported in [4]-[6], there is a physical risk of Loss of Synchronism (LOS) of the WTs during severe voltage dips, where the grid voltage at the point of connection ( $\mathrm{PoC}$ ) of a WPP drops to very low values, e.g. below $0.2 \mathrm{pu}$. During this severe drop down to very low values, the grid side converters of the WTs experience the LOS as a physical fact [4]-[6]. Since there is no valid operating point for the referenced active and reactive currents with this very low grid voltage, the WTs may lose their synchronism with the grid fundamental frequency and lose the control of active and reactive currents. Furthermore, the LOS causes power system simulations non-convergence errors for the rms simulations.

There are few studies in the literature to solve the LOS problem of the WTs. These methods are briefly reviewed here only for the sake of coherency, though solving the LOS is not the main scope of this paper. In [5], [6] and [7], a voltage dependent active current reduction method during low voltage faults is suggested to avoid LOS. Assuming that the impedance between the WT terminal and the faulted grid point around $\mathrm{PoC}$, is highly inductive (high $\mathrm{X} / \mathrm{R}$ ratio), it helps to avoid the LOS to reduce the active current reference as voltage goes down. In [6], a novel method of freezing the calculated phase angle when the voltage drops down to very low values is suggested. Thus, the deviation of angle and frequency is avoided artificially keeping the angle constant; in other words renouncing the grid synchronization. As a result, the active and reactive current of the WT flows in accordance with the X/R of the impedance. In [4], another novel method called PLL frequency based active current injection is proposed to solve the LOS problem in a closed loop control manner, where the active current is modified accordingly if the frequency of the WT terminal voltage is detected to deviate from rated due to the LOS. In [8], the LOS is tried to be avoided through the calculation of the phase angle at the converter connection terminals and controlling the power injection, where such an idea can be further improved using PMU data from remote locations. In addition 
This article has been accepted for publication in a future issue of this journal, but has not been fully edited.

Content may change prior to final publication in an issue of the journal. To cite the paper please use the doi provided on the Digital Library page.

to these, there might be additional methods to avoid LOS and also other possible instabilities, however as mentioned above, solving the LOS is not the main focus of this paper. As it will be shown in the following sections, the above-mentioned LOS problem for the WTs appears as non-convergence errors within the rms simulations, since the network solver cannot reach any grid solution point at the end of iterations, as expected if the grid synchronization dynamics is omitted (i.e. instantaneous angle calculation or ideal synchronization). The abovementioned LOS solution methods are summarized in Table I, whereas this paper handles the non-convergence problem of the rms simulations during the LOS, for the first time in the literature.

Table 1 Comparison of LOS solutions from literature

\begin{tabular}{ll}
\hline PLL frequency based active current reduction [4] & $\begin{array}{l}\text { Full utilization of current capacity with } \\
\text { grid code compliance } \\
\text { Extensive tuning requirement }\end{array}$ \\
Voltage dependent active current reduction [5] & $\begin{array}{l}\text { Not fully used current capacity } \\
\text { No guarantee for grid code compliance }\end{array}$ \\
& $\begin{array}{l}\text { PLL blocking during the active current } \\
\text { reduction } \\
\text { Active current reduction with angle freezing [6] }\end{array}$ \\
& No guarantee for grid code compliance \\
\hline
\end{tabular}

In this paper, as a solution to the simulation convergence problem in rms simulations with the conventional method mentioned above as the ideal grid synchronization, a first order low pass filter is proposed to represent the phase angle calculation for the WT's current injection. Accordingly as discussed and presented by simulations, this solution is sufficient enough in the rms simulations for the calculation of the phase angle without any convergence errors and the clear presentation of the LOS problem. In the following section, the conventional method of instantaneous phase angle calculation (i.e. ideal grid synchronization approach) is given as a reference with a non-severe fault example, where the grid voltage drops to 30 per cent, and it is shown that the classical approach works properly with non-severe voltage dips. Accordingly, the severe short-circuit fault example with 0.1 per cent remaining voltage, which causes the LOS and convergence problems is presented in the second section. The proposed first order low pass filter approach and its effect on the simulation convergence are introduced in the third section and the proposed solution is compared with a PI based PLL method. The results are summarized and discussed in the fourth section with further suggestions to system operators and power system tool developers. 
This article has been accepted for publication in a future issue of this journal, but has not been fully edited.

Content may change prior to final publication in an issue of the journal. To cite the paper please use the doi provided on the Digital Library page.

\section{Instantaneous Angle Calculation In RMS Models}

In power system analysis, within the rms simulations the terminal voltage phase angle of the gridconnected converter (in this case the WT grid side converter) is calculated directly from the WT terminal voltage phasor available as below.

$$
\cos p h i=\frac{\operatorname{Re}(u)}{|u|} \quad \text { and } \quad \sin p h i=\frac{\operatorname{Im}(u)}{|u|}
$$

where $u$ is the terminal voltage of the WT terminal and phi is the calculated voltage phase angle (positive sequence). This phase angle is calculated using the common (grid) reference frame from the voltage phasor representation (re-im axes) as stated above. This approach, which is not making use of any grid synchronization algorithm and calculating the actual phase angle without a delay, is named as 'instantaneous angle calculation' in this study. Furthermore, in the converter model [1], the reference currents (Idref and Iqref in the control $(d-q)$ reference frame) are converted and injected as real and imaginary currents (Ire and Iim) to the grid using this calculated phase angle.

A test network, which is shown in Figure 1, is used for the simulation studies in this paper. The WPP is aggregated as a single WT model and connected to a Thevenin equivalent grid composed of a WT transformer (between Bus5-Bus4), a collector system, a WPP transformer (between Bus3-Bus2) and connection lines of the WPP (between Bus2-Bus1). The collector system which is the MV feeders of the WPP is also aggregated as a single cable (between Bus4-Bus3) as shown in Figure 1. Simulations in this and the following sections are conducted using DIgSILENT PowerFactory simulation software. The details of this network model are provided in Table A-I in the appendix. It is worth noting that the X/R ratio for the total impedance between the WT terminal (Bus5) and the PoC (Bus2) is around 11.

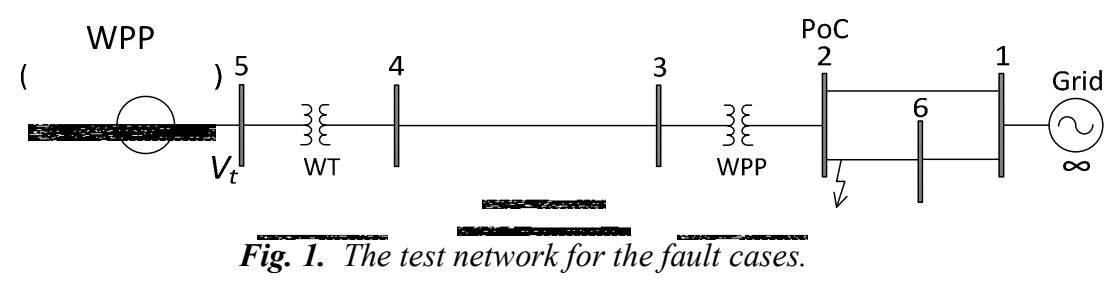

\subsection{Performance of Instantaneous Angle Calculation during Non-Severe Voltage Dips}

In order to present the performance of the instantaneous angle calculation method as a reference, a non-severe fault is created at the line between Bus 2 and Bus 6. The remaining voltage at the PoC is 30 per cent (Figure 2-a), and due to the low voltage (as regards the grid code [3]) the reactive current (Iq) 
This article has been accepted for publication in a future issue of this journal, but has not been fully edited.

Content may change prior to final publication in an issue of the journal. To cite the paper please use the doi provided on the Digital Library page. reference increases to $1 \mathrm{pu}$ and the active current $(I d)$ reference, which is $1 \mathrm{pu}$ before the fault, decreases down to $0.45 \mathrm{pu}$ during the fault as observed in Figure 2-b and 2-c.

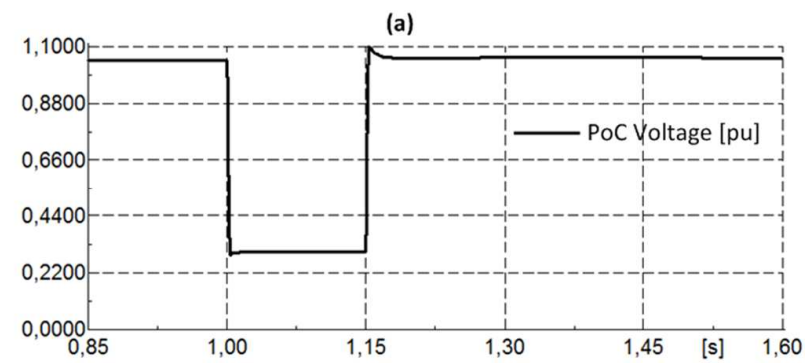

(b)

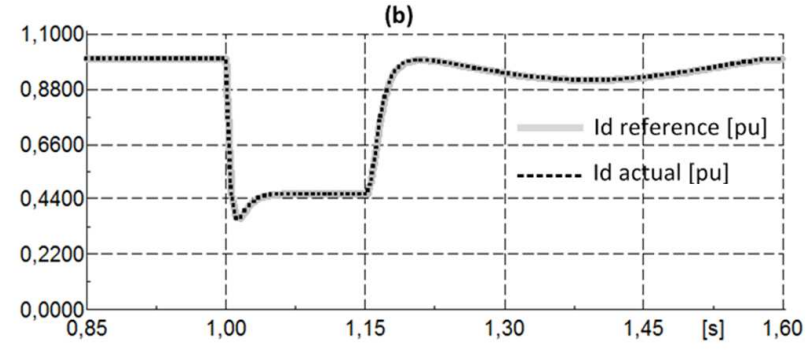

(c)
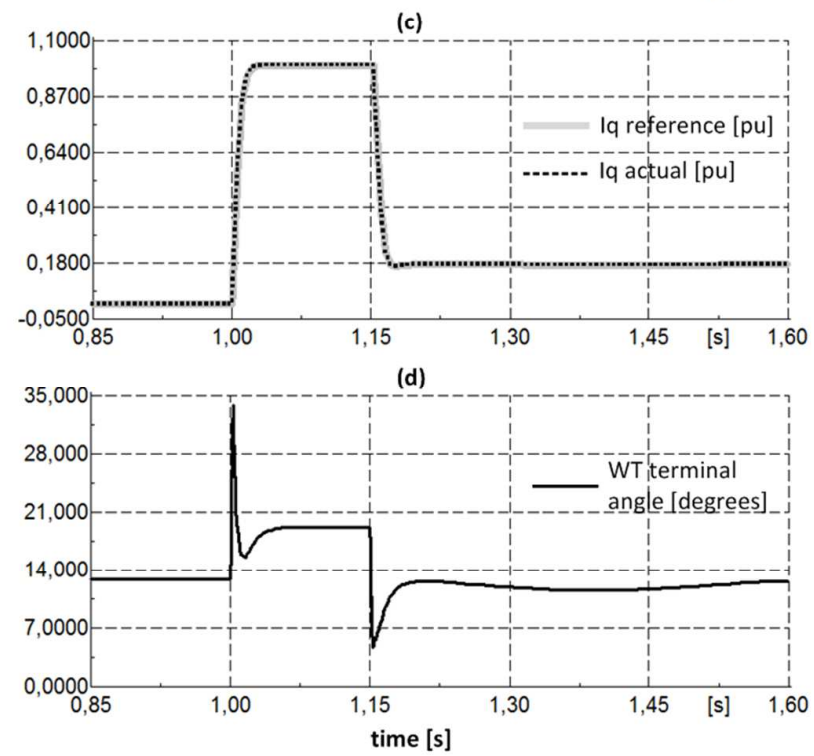

Fig. 2. Non-severe (VPoC=30\%) fault (a) VPoC [pu] (b) active current (Id) reference (solid gray) and actual (dotted black) [pu] (c) reactive current (Iq) reference (solid gray) and actual (dotted black) [pu] (d) WT terminal voltage angle [degrees].

The terminal phase angle in Figure 2-d, which is instantaneously calculated by the network solver, is also used by the converter during current injection. Hence the active and reactive current references are ideally realized without any error as observed in Figure 2-b and 2-c. As presented in the figures the rms simulations with the conventional method of the instantaneous angle calculation perform sufficiently during non-severe faults. The severe voltage dips, which cause the LOS and convergence problems, are investigated in the following sub-section. 
This article has been accepted for publication in a future issue of this journal, but has not been fully edited.

Content may change prior to final publication in an issue of the journal. To cite the paper please use the doi provided on the Digital Library page.

\subsection{Convergence Problem during Severe Voltage Dips}

As stated in the grid codes [3], as similar to conventional power plants, WPPs are also requested to withstand solid faults with 0 per cent remaining voltage at their connection points. A solid short-circuit fault at the line between Bus 2 and Bus 6, and very close to the Bus 2 end is simulated, where the PoC voltage drops down to 0.1 per cent. The WPP is referenced to inject 1 pu reactive current and $0.45 \mathrm{pu}$ active current (remaining capacity) during the fault, as observed in Figure 3-b and 3-c.

Due to the LOS occurrence, the active and reactive current references cannot be realized. Uncontrolled high currents flow and the simulation results in no convergence error since there is no valid operating point for these references. The phase angle is calculated erroneously, hence the injected currents ( $I_{d}$ and $I_{q}$ which are based on the incorrect phase angle) are not composed of the referenced active and reactive components. Accordingly, the voltage magnitude and phase angle at WT terminal, which are calculated at each simulation step by the network solver, cannot flow the reference currents correctly to the PoC. There is no network solution due to the LOS, and furthermore the impact of the instantaneous angle calculation on the simulation is the no convergence errors. Moreover, the LOS occurs when the WT is referenced to inject inconvenient active and reactive currents, which cannot be transferred to the faulted (very low voltage) grid through the impedance between the WT and the PoC [4]-[6]. As a result of nontransferrable current references and the instantaneous angle calculation, the network solver of the rms simulation cannot converge to a valid operating point and produces convergence errors, which perturb the simulation.

As shown by simulations in [6] and also by experiments in [4], for the WT converters employing a (PI-based) PLL algorithm, the LOS during very low voltage faults causes the PLL to become unstable and consequently substantial frequency deviation and angle slippage are observed. However as observed here, for the rms simulation, where the PLL is not modelled, the LOS causes the simulation non-convergence problem, since the solver cannot reach any quasi steady-state operating point at the end of each iteration, as expected. In order to avoid ambiguity, it should be noted that the abovementioned LOS and nonconvergence problems are also observed as the same within the simulations using the Eurostag software.

Considering the large-scale simulations, for instance the Pan-European power system, the described non-convergence problem of the WPPs would result in serious difficulties in terms of robustness and performance of the simulations. Hence, to avoid the non-convergence problem the first order low-pass filter (LPF) based angle calculation solution is proposed in the following section. 
This article has been accepted for publication in a future issue of this journal, but has not been fully edited.

Content may change prior to final publication in an issue of the journal. To cite the paper please use the doi provided on the Digital Library page.

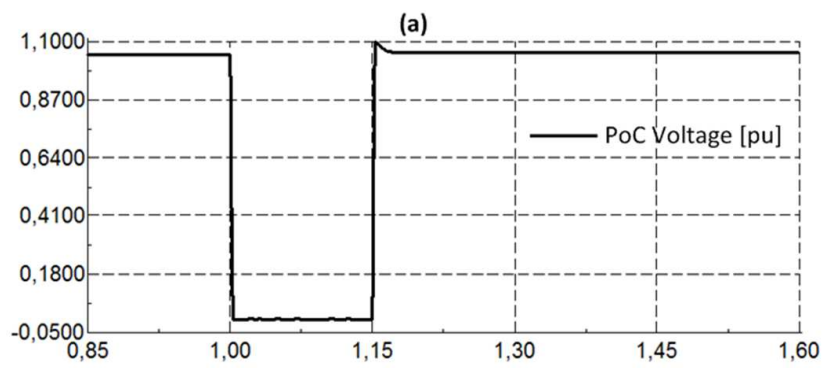

(b)

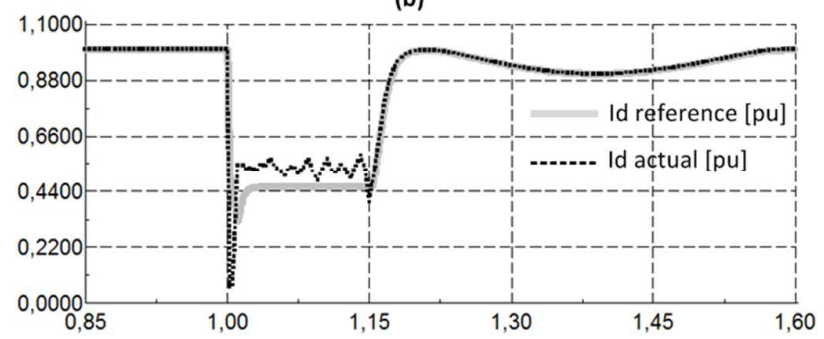

(c)

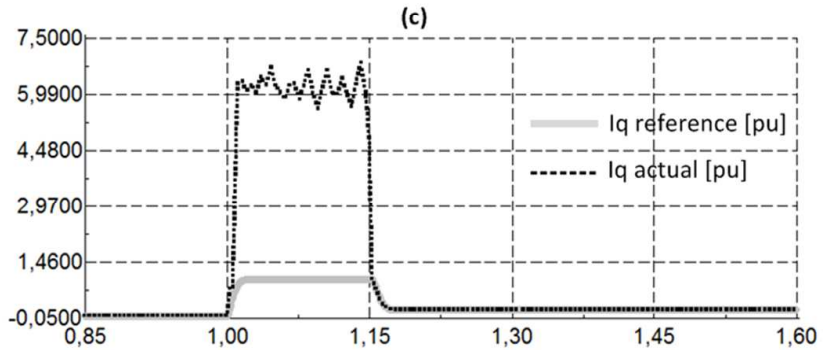

(d)

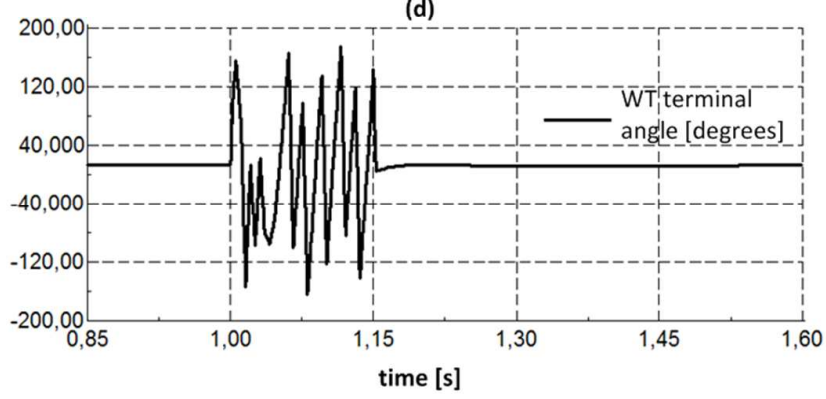

Fig. 3. (LOS and convergence problems) during severe $\left(V_{P o C}=0.1 \%\right.$ ) fault. (a) $V_{P o C}[p u]$ (b) active current (Id) reference (solid gray) and actual (dotted black) [pu] (c) reactive current (Iq) reference (solid gray) and actual (dotted black) [pu] (d) WT terminal voltage angle (wrap between \pm 180 ) [degrees].

\section{Proposed LPF Based Angle Calculation}

As given in the second section, the method of instantaneous angle calculation works as an ideal grid synchronization algorithm via the calculation of the phase angle directly from the WT terminal voltage phasor. As shown in the previous section, with the ideal instantaneous angle calculation, the rms simulation generates non-convergence errors when the non-transferrable current references are tried to be injected during very low voltage faults. However, in real WT converter implementation, grid synchronization algorithms (e.g. PI based PLL or first order filter) are employed, which introduce delays and hence non-ideal responses [9]. These grid synchronization algorithms generally have time constants 
This article has been accepted for publication in a future issue of this journal, but has not been fully edited.

Content may change prior to final publication in an issue of the journal. To cite the paper please use the doi provided on the Digital Library page.

lower than $1 \mathrm{msec}$. However, these time constants cannot be implemented to represent the grid synchronization dynamics in the rms simulations where the integration time step is limited to $1 / 4$ of the fundamental cycle (i.e. $5 \mathrm{msec}$ ) [1]. In order to represent the grid synchronization dynamics in a realistic way and without adding complexity, a LPF is proposed as an angle calculation method, as shown in Figure 4. The phase angle is measured at the WT terminal, filtered by the first order LPF, and then cosphi and sinphi signals are sent to the reference frame rotation as inputs. The proposed LPF filter introduces a time delay for the angle calculation; hence the grid synchronization response for current injection is dynamically slowed down and is not ideal anymore. As it can be seen later in this section, with the use of the LPF, the simulation non-convergence problem is overcome; however the LOS still exists, as expected. Due to the LOS, the current references are determined by the X/R ratio of the impedance between the WT and the fault location, as explained in [4] and [6], and the frequency and angle deviations are observed. It should be noted that the proposed LPF provides simulation convergence, without focusing on solving the LOS problem.

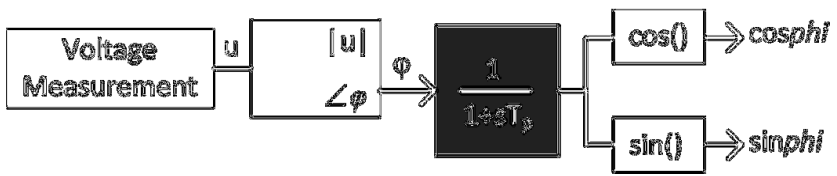

Fig. 4. The proposed first order Low-Pass Filter (LPF) based angle calculation solution to avoid simulation non-convergence problem.

The proposed LPF also stands as a generic grid synchronization method, as having the characteristics of an arctangent and filtering based synchronization method [9]. In comparison to a higher order grid synchronization method, such as a PI based PLL, the proposed LPF method is computationally feasible for the rms simulations. For instance while the PI based synchronous frame PLL requires tuning of PI parameters, saturation limits, and anti-windup mechanisms [10] - [11], the LPF requires tuning of only one single parameter, the filter time constant $T_{p}$. Comparison of the proposed LPF dynamics against a PI-based PLL method during a generic phase jump is shown in Figure 5. As seen in Figure 5, the LPF provides a good approximation for the dynamics of the PI-based PLL, solving the non-convergence problem during very low voltage faults (Figure 6), without adding significant complexity. As reported in [4] and [6], the steady-state phase angle error occurs during the LOS causing fault, while the error here helps the simulation solver to reach convergence but without realizing the references, as they are nontransferrable. 
This article has been accepted for publication in a future issue of this journal, but has not been fully edited.

Content may change prior to final publication in an issue of the journal. To cite the paper please use the doi provided on the Digital Library page.

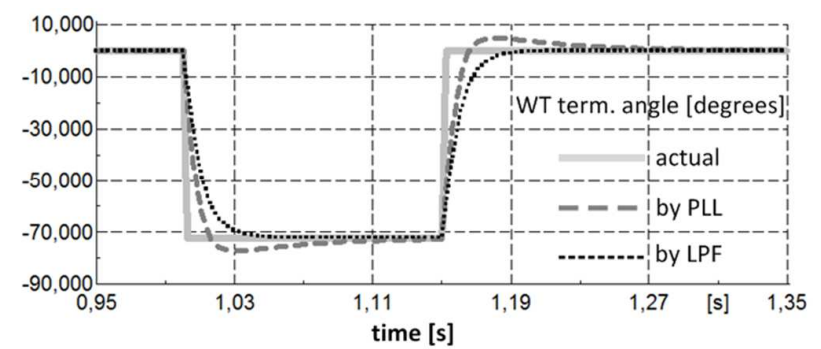

Fig. 5. Comparison of angle tracking dynamics for a generic phase jump case; terminal actual phase angle (solid gray), PLL calculated angle (dashed gray), LPF calculated angle (dotted black) [degrees].

In Figure 6, results during the severe voltage dip, which causes LOS, are given both for the proposed LPF based solution and PI-based PLL implementation. In Figure 6, the response of the WT with the proposed LPF is a good approximation of the PI-based PLL, with less complexity. As observed in Figure 6-b and 6-c, since the PoC voltage is very low ( 0.1 per cent) and the WT terminal voltage magnitude is $0.18 \mathrm{pu}$, the actual active and reactive currents are determined by the $X / R$ ratio of the impedance (between the WT terminal and the PoC). Regardless of the current references (output of the WT FRT control), the actual active current is $0.1 \mathrm{pu}$ and the actual reactive current is $1.09 \mathrm{pu}$ during the fault; such that the $I q / I d$ ratio (10.9) is consistent with the $X / R$ ratio of the impedance between the WT terminal and the fault location.

Due to the LOS, the steady-state phase angle error occurs, which causes the frequency deviation and hence phase angle slippage as in Figure 6-d. It should be noted that the LOS occurs as in Figure 3 but without the simulation non-convergence errors in this case. The response time of both methods improves the convergence and helps the network solver. The calculated phase angle in Figure 6 has more realistic dynamics than the instantaneous calculation. Nevertheless, it is valuable that with the LPF method; it is still possible to observe that the frequency is deviating, phase angle at WT terminals occurs to run asynchronously against the grid voltage phasor, and current control is lost (active and reactive current references are not realized exactly). Hence, the solutions for LOS can be implemented further by the model developers and manufacturers using the LPF solution.

The three main benefits of the LPF method can be summarized as; simulation convergence for very low voltage faults (even for 0 per cent faults at $\mathrm{PoC}$ ), representation of grid synchronization dynamics with minimum additional complexity, and possibility to observe the LOS occurrence, which gives the inputs to solve it separately. 
This article has been accepted for publication in a future issue of this journal, but has not been fully edited.

Content may change prior to final publication in an issue of the journal. To cite the paper please use the doi provided on the Digital Library page.

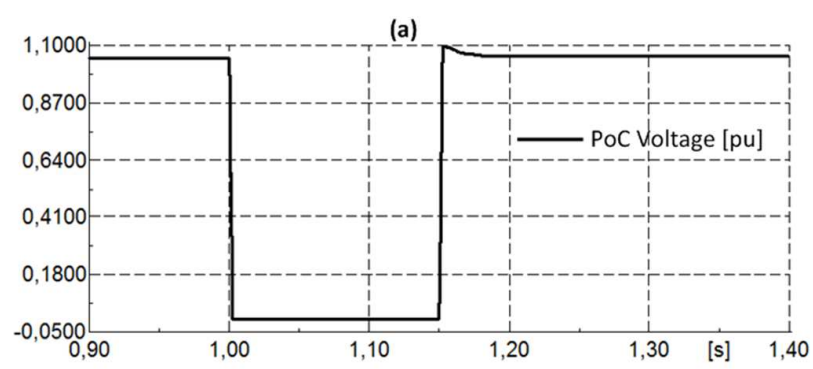

(b)

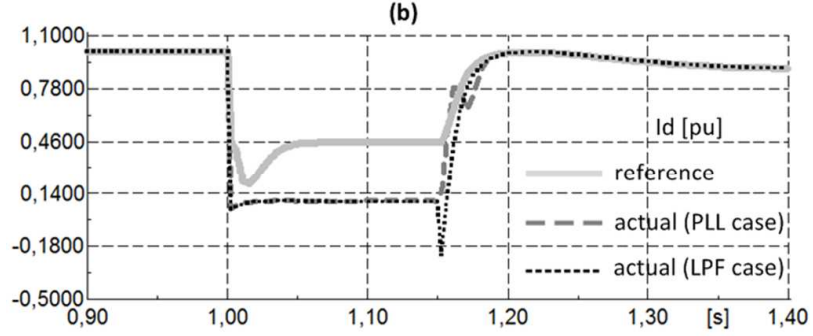

(c)

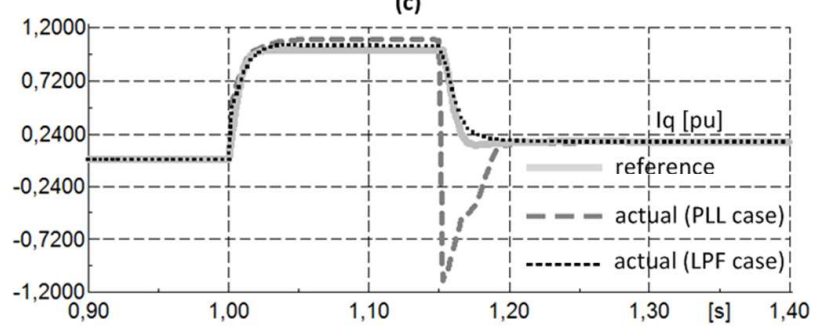

(d)

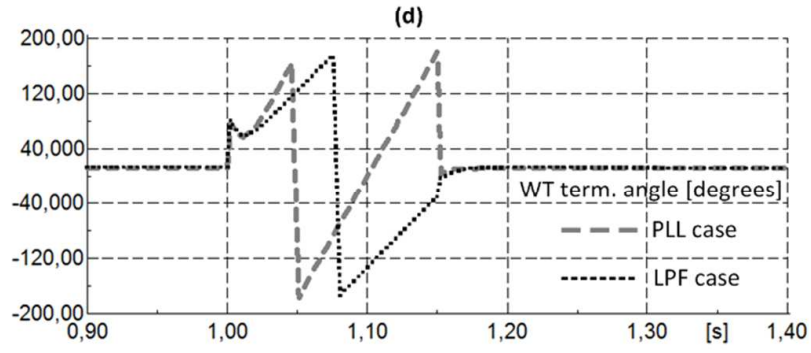

Fig. 6. Simulation results with a PI-based PLL method and the proposed LPF method, during a severe fault (Vpoc=0.1\%) (a) Vpoc [pu] (b) active current (Id) reference (solid gray), actual in PLL case (dashed gray) and actual in LPF case (dotted black) [pu] (c) reactive current (Iq) reference (solid gray), actual in PLL case (dashed gray) and actual in LPF case (dotted black) [pu] (d) WT terminal voltage angle (wrap between \pm 180 ) in PLL case (dashed gray) and in LPF case (dotted black) [degrees].

As seen in Figure 5, the LPF based angle calculation causes a delay in the phase angle tracking dynamics, which can impair the dynamic performance of WT's active and reactive current injection, also during the non-faulty steady-state conditions. This delay can influence response of the WT within its fundamental control loops, e.g. voltage control. It should be noted that the delay and hence its impact can be minimized by reducing the time constant of the LPF, however this would need a smaller simulation step size (e.g. less than $1 \mathrm{~ms}$ ), compromising the simulation time of large power systems. Considering the suggested minimum integration step size of $5 \mathrm{~ms}$ for the rms simulations, the delay caused by the LPF 
This article has been accepted for publication in a future issue of this journal, but has not been fully edited.

Content may change prior to final publication in an issue of the journal. To cite the paper please use the doi provided on the Digital Library page. cannot be decreased below 10ms [1]. In order to avoid the impact of the LPF during non-faulty steadystate conditions, a triggering mechanism is implemented, which activates the LPF only when there is a severe voltage dip, in other words only when there is a risk of LOS and the simulation non-convergence. As presented in Figure 7, the WT terminal voltage phasor angle is filtered only when the WT terminal voltage magnitude drops below a threshold value, $u_{t h}$ which can be set as $0.4 \mathrm{pu}$. Moreover, employing the triggering mechanism also allows using a larger time constant (e.g. 100ms) for the LPF filter such that the deviation of angle during LOS is further slowed down and more stable simulation is obtained. The results of the proposed LPF based angle calculation with the threshold triggering function are given in Figure 8.

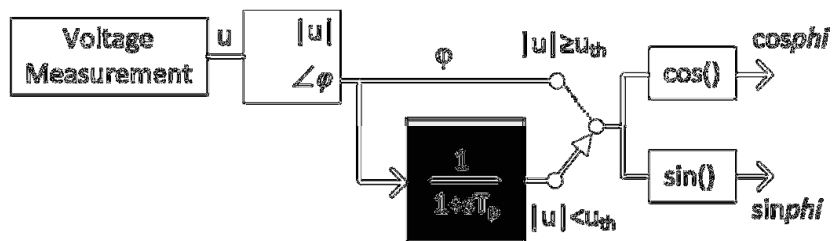

Fig. 7. The proposed first order LPF based angle calculation with the threshold triggering function to avoid simulation nonconvergence problem during severe faults.

The overshoots of active and reactive currents in Figure 6 immediately after the fault clearance are avoided by the threshold triggering function (Figure 7), as seen in Figure 8. The proposed triggering function performs adequately during the post-fault period for rms simulations, since the model switches to ideal instantaneous phase angle calculation immediately when the voltage recovers with fault clearance.

The proposed LPF method provides robustness for $\mathrm{rms}$ simulations whenever a coordinate transformation is performed. The proposed LPF model as a phase angle calculation method is proposed to be used within rms simulation of not only type 4 WT models of IEC61400-27-1 but also other type 4 models of other developers [2], type 3 (DFIG-based) models in [1], and for models of other currentcontrolled converter-based grid-connected units (e.g. STATCOM and HVDC). 
This article has been accepted for publication in a future issue of this journal, but has not been fully edited.

Content may change prior to final publication in an issue of the journal. To cite the paper please use the doi provided on the Digital Library page.
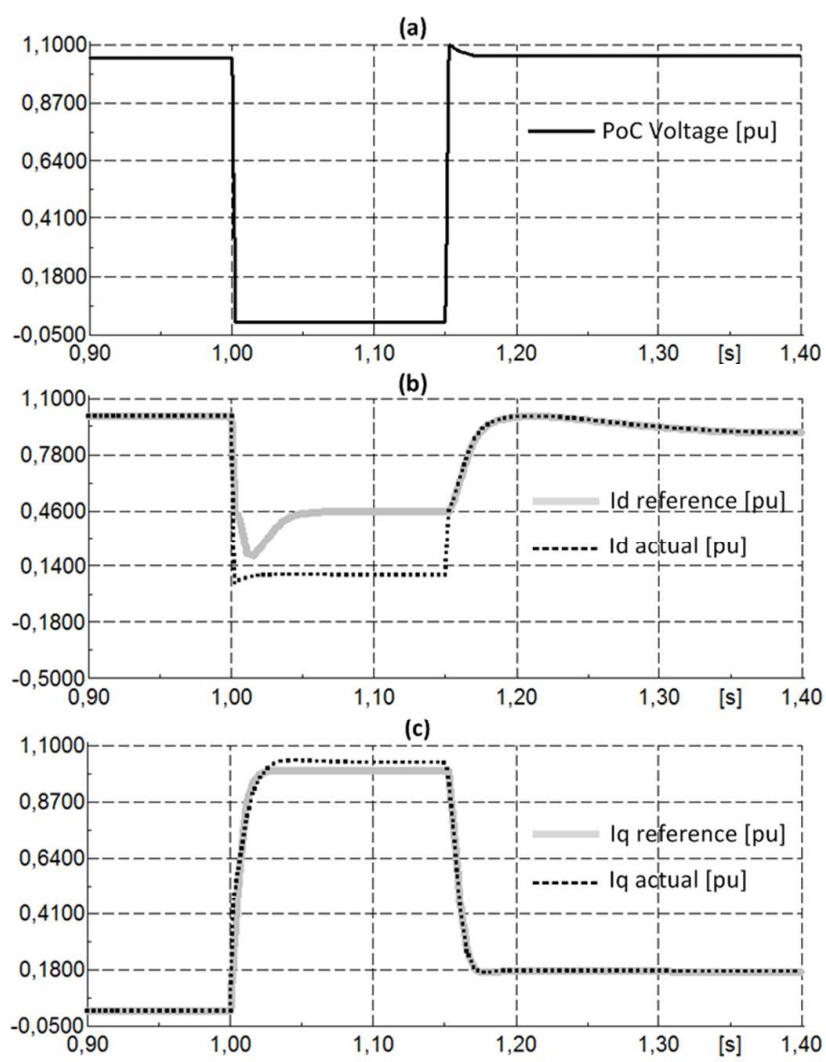

(d)

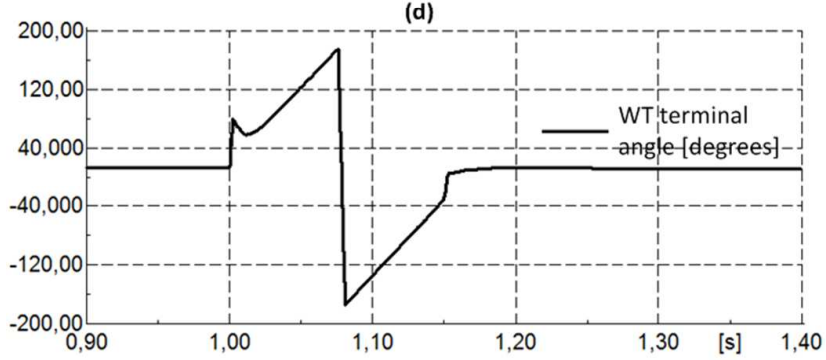

Fig. 8. Simulation results of the proposed LPF method with threshold triggering function during a severe fault (Vpoc=0.1\%) (a) Vpoc [pu] (b) active current (Id) reference (solid gray) and actual (dotted black) [pu] (c) reactive current (Iq) reference (solid gray) and actual (dotted black) [pu] (d) WT terminal voltage angle (wrap between \pm 180$)$ [degrees].

\section{Results \& Discussion}

The proposed LPF method solves the rms simulation non-convergence problem arising due to the LOS during very low voltage faults. With the implementation of the LPF method, the simulation results in Figure 6 and Figure 8 have better convergence performance and can present the LOS occurrence more clearly compared to Figure 3. Therefore, the LPF based phase angle calculation method provides simulation stability during the LOS and can be included within the WT modelling standards [1]. Additionally, the calculated phase angle dynamics represent a realistic behaviour which allows the developers to implement and present their specific solutions for the LOS problem to the system operators. 
This article has been accepted for publication in a future issue of this journal, but has not been fully edited.

Content may change prior to final publication in an issue of the journal. To cite the paper please use the doi provided on the Digital Library page.

In order to avoid the LOS occurrence (i.e. not a solution to the problem) in WPP integration studies, it might be considered to conduct short-circuit fault studies where the PoC voltage of the WPPs are not reduced down to very low voltage values (i.e. above $0.2 \mathrm{pu}$ ). However, in this case the analysis of severe voltage faults (e.g. a solid three-phase fault), which are required by the grid codes today [3], are compromised which might need further discussions on necessity of these fault tests for WPPs. In [4] and [5], it is shown that if the remaining PoC voltage larger than the magnitude of the impedance between the WT terminal and the WPP PoC in per unit, the LOS occurrence can be avoided. Hence, the fault studies with a fault impedance or using a remote fault location from the $\mathrm{PoC}$ can be considered as a suggestion to the system operators for the future grid codes.

As an alternative to the proposed LPF solution, a high order grid synchronization method (e.g. PIbased PLL) can still be implemented to avoid non-convergence problems, but compromising the simplicity of the rms simulation compared to the LPF method. Moreover, tuning of PLL is critical in terms of the low-frequency large signal instability of the converters, which are connected to weak grids (i.e. high impedance connection) [12]. The proposed LPF as a grid synchronization has the potential to be used for power system stability analysis of the weak grid (high impedance) connections of WTs, which stands as a future work.

In accordance with the increasing share of WPPs in the power system, WPPs are involved in provision of ancillary services, such as power oscillation damping control. In case of a converter simulation with current control along with grid synchronization, small-signal stability analysis should be conducted to tune the controllers properly and to observe impact of the grid synchronization dynamics on the electromechanical oscillations with such ancillary services from the WPPs.

\section{Conclusion}

Owing to the large share of wind power in power systems of certain countries, especially in Europe, the need of power system analysis with WPPs arises. WT and WPP models have been developed by the academia and WT manufacturers, which are chosen as rms models in order to provide computational simplicity and speed, considering large-scale simulations. Additionally, standards for the developed WT and WPP models have been developed by IEC and WECC working groups. As a common requirement of the grid codes, the developed models are utilized for short term voltage stability and fault ride-through studies. The conventional method of instantaneous phase angle calculation, i.e. ideal grid synchronization, performs well with moderately low voltage faults. However, it is shown that the physical fact of the LOS of WT converters during severe voltage dips is observed to cause simulation non-convergence problems with the instantaneous angle calculation method in rms simulations. 
This article has been accepted for publication in a future issue of this journal, but has not been fully edited.

Content may change prior to final publication in an issue of the journal. To cite the paper please use the doi provided on the Digital Library page.

In this paper, a first order low-pass filter (LPF) is proposed as angle calculation method in converterbased WTs in order to solve the non-convergence errors during severe voltage faults. The proposed LPF method is shown in order to solve the non-convergence errors even for solid faults (i.e. a zero impedance three-phase fault). Additionally, implementing a threshold triggering function to activate the LPF based calculation only during severe voltage dips avoided any possible impairment during healthy steady-state operations. Moreover, the frequency deviation due to the LOS problem, which needs to be solved with additional control methods, is shown to be represented with the LPF method. Hence, the proposed LPF method gives the possibility to detect if the WPP experiences the LOS and hence to solve it.

The recently published IEC 61400-27-1 wind turbines electrical simulation models standard [1] has adopted the proposed idea of utilizing a first order low-pass filter as phase angle calculation in rms simulations, which helps to obtain robust simulations, especially when a large scale (e.g. Pan-European) power system with high share of wind power is simulated and analysed.

\section{Acknowledgments}

This work was supported by the European Commission through the European FP7 project iTesla under the grant agreement no 283012.

\section{References}

[1] IEC61400-27-1 Ed. 1 'Wind Turbines - Part 27-1: Electrical simulation models - Wind Turbines', Feb. 2015.

[2] Asmine, M., Brochu, J., Fortmann, J., et al.: 'Model Validation for Wind Turbine Generator Models, ' IEEE Transactions on Power Systems 2011, 26, (3), pp.1769-1782.

[3] ENTSO-E: 'Network Code for Requirements for Grid Connection Applicable to all Generators' (NC RfG), European Network of Transmission System Operators for Electricity ENTSO-E, 2015.

[4] Goksu, O., Teodorescu, R., Bak, C. L., Iov, F., Kjaer, P. C.: 'Instability of Wind Turbine Converters During Current Injection to Low Voltage Grid Faults and PLL Frequency Based Stability Solution, ' IEEE Transactions on Power Systems 2014, 29, (4), pp.1683-1691.

[5] Erlich, I., Shewarega, F., Engelhardt, S., Kretschmann, J., Fortmann, J., Koch, F.: 'Effect of wind turbine output current during faults on grid voltage and the transient stability of wind parks,' IEEE Power and Energy Society General Meeting, July 2009, pp. 1-8.

[6] Weise, B.: "Impact of K-factor and active current reduction during fault-ride-through of generating units connected via voltage-sourced converters on power system stability,' IET Renewable Power Generation 2015, 9, (1), pp. 25-36.

[7] Fortmann, J. Engelhardt, S., Kretschmann, J., Feltes, C., Erlich, I.: 'New Generic Model of DFG-Based Wind Turbines for RMS-Type Simulation,' IEEE Transactions on Energy Conversion 2014, 29, (1), pp. $110-118$.

[8] Larsen, E. V., Klodowski, A. M., and S. A. Barker: 'Method and Systems for Operating a Wind Turbine, ' US 8,046,109 B2, Oct. 25, 2011.

[9] Blaabjerg, F., Teodorescu, R., Liserre, M., Timbus, A. V.: 'Overview of Control and Grid Synchronization for Distributed Power Generation Systems," IEEE Transactions on Industrial Electronics 2006, 53, (5), pp.1398-1409.

[10] Yazdani, A. and Iravani, R.: 'Voltage-Sourced Converters in Power Systems, ' IEEE/Wiley, 2010.

[11] Teodorescu, R., Liserre, M., Rodriguez, P.: 'Grid converters for photovoltaic and wind power systems, ' Wiley Press, 2011. 
This article has been accepted for publication in a future issue of this journal, but has not been fully edited.

Content may change prior to final publication in an issue of the journal. To cite the paper please use the doi provided on the Digital Library page.

[12] Dong, D., Bo, W., Boroyevich, D. Mattavelli, P., Yaosuo, X.: 'Analysis of Phase-Locked Loop LowFrequency Stability in Three-Phase Grid-Connected Power Converters Considering Impedance Interactions,' IEEE Transactions on Industrial Electronics 2015, 62, (1), pp.310-321.

\section{Appendix}

Table A-1 Test Network Data (Figure 1)

\begin{tabular}{lc}
\hline Base Power & $100[\mathrm{MVA}]$ \\
Grid Voltage & $230[\mathrm{kV}]$ \\
WPP PoC Voltage & $34,5[\mathrm{kV}]$ \\
WT Terminal Voltage & $0.6[\mathrm{kV}]$ \\
WT Transformer & $\mathrm{j} 0.05[\mathrm{pu}]$ \\
Collector equivalent & $0.015+\mathrm{j} 0.025[\mathrm{pu}]$ \\
WPP Transformer & $\mathrm{j} 0.1[\mathrm{pu}]$ \\
Line 2-1 & $0.01+\mathrm{j} 0.1[\mathrm{pu}]$ \\
Line 2-6 & $0.005+\mathrm{j} 0.05[\mathrm{pu}]$ \\
Line 6-1 & $0.005+\mathrm{j} 0.05[\mathrm{pu}]$ \\
Fault Impedance & $\mathrm{j} 10[\Omega]$ \\
\hline
\end{tabular}

